\title{
Hyperlactatemia in a group of HIV patients living in Yaounde-Cameroon
}

Corinne Tchoula Mamiafo ${ }^{3}$, Vicky Jocelyne Ama Moor ${ }^{1,2^{*}}$, Jobert Richie N Nansseu ${ }^{4}$, Constant Anatole Pieme ${ }^{2}$, Claude Tayou ${ }^{5}$ and Jeanne Ngogang Yonkeu ${ }^{1,2}$

\begin{abstract}
Background and aim: Prolonged exposure to highly active antiretroviral therapy (HAART) is associated with adverse effects such as hyperlactatemia. We determined the prevalence and risk factors for developing hyperlactatemia among human immunodeficiency virus (HIV)-infected cameroonians on antiretroviral therapy (ART).

Methods: We conducted a cross-sectional study from January to April 2012 involving 91 HIV-infected patients receiving ART for at least 12 months and $30 \mathrm{HIV}$-infected patients who have never received ART (ART-naïve patients). Plasma lactate levels were determined after at least 12 hours of overnight fasting and hyperlactatemia defined as lactate concentrations $\geq 3 \mathrm{mmol} / \mathrm{L}$. The prevalence of hyperlactatemia was determined and the risk factors were analyzed by a multivariate logistic regression model.

Results: The mean lactataemia was significantly higher in the group of HIV patients currently taking ART than in the ART-naïve one $(2.3 \pm 1.3$ and $1.7 \pm 0.7 \mathrm{mmol} / \mathrm{L}$ respectively, $p=0.002)$. Patients on first line ART regimens had significantly higher lactatemia than those on second line regimens $(2.5 \pm 1.5$ and $1.9 \pm 0.7 \mathrm{mmol} / \mathrm{L}$ respectively, $p=0.014)$. The prevalence of hyperlactatemia in HIV patients receiving ART and in ART-naïve HIV patients was respectively 18.7 and 6.7\% ( $p=0.095)$. ART-exposure (adjusted odds ratio (aOR) 5.44, 95\% confidence interval $(\mathrm{Cl})$ $1.06-27.84 ; p=0.042)$ and being on a first line regimen ( $a O R$ 16.22, 95\% Cl $1.57-167.91 ; p=0.019$ ) were independent strong predictors of hyperlactatemia.

Conclusion: Hyperlactatemia was not rare in our study population. Being on a first line regimen constitutes an important risk factor for developing hyperlactatemia. Measurement of plasma lactate may be useful in optimizing the management of HIV-positive persons on ART.
\end{abstract}

Keywords: Hyperlactatemia, Antiretroviral therapy, HIV, First line regimen

\section{Introduction}

AIDS remains one of the major public health hazards in Africa. The advent and widespread use of HAART in clinical practice has profoundly modified the natural history of HIV infection by significantly improving the prognosis and quality of life of people living with the infection, with a drastic reduction in mortality and morbidity related to HIV and its complications [1]. Unfortunately,

\footnotetext{
* Correspondence: movicky@yahoo.fr

'Laboratory of Biochemistry, Yaounde University Teaching Hospital, Yaounde, Cameroon

${ }^{2}$ Department of Biochemistry and Physiological Sciences, University of Yaounde I, Yaounde, Cameroon

Full list of author information is available at the end of the article
}

the need for permanent medication has led to increasingly more numerous descriptions of new adverse metabolic effects, this being explained by mitochondrial toxicity [2]. It has been demonstrated that this mitochondrial toxicity is associated with hyperlactatemia, lactic acidosis, hepatic steatosis, pancreatitis, lipodystrophy, and peripheral neuropathy [3].

Hyperlactatemia characterized by mild to moderate increases in blood lactate levels in the absence of acidosis has been reported in $8-36 \%$ of patients receiving HAART in developed countries, although the majority of cases are asymptomatic or have very mild symptoms [4-8]. Only a small number of patients develop the most severe form of hyperlactatemia, which

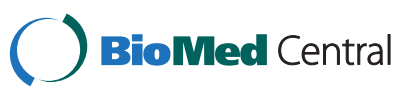


presents as metabolic acidosis and is associated with a high mortality rate.

A variety of NRTIs, including stavudine, zidovudine and didanosine, have been associated with hyperlactatemia because of their potential for mitochondrial toxic effects [2,6,9-14]. While stavudine is rarely used in resource-rich settings and is no longer recommended by WHO for initial treatment of HIV-1 infection [14], it remains an important component of standard ART regimens in many resource-limited countries, mainly due to its lower cost $[15,16]$. Some observational studies have suggested that specific risk factors associated with the development of hyperlactatemia include female sex [17-23], elevated weight or BMI [17,20-23], older age (> 40 years) [17,20], and lower CD4 cell counts [17].

There are limited data on hyperlactatemia caused by prolonged exposure to ART in resource-limited settings like Cameroon. The present study sought at determining the prevalence and determinants related to the development of hyperlactatemia in HIV-infected Cameroonians currently on ART.

\section{Patients and methods \\ Study participants}

This cross-sectional survey was carried out at the HIV out-patient clinic of the Yaounde University Teaching Hospital and the Yaounde Central Hospital. These centers are located in the political capital of Cameroon, and receive a large number of HIV-infected patients living in Yaounde and surrounding town. A convenient sample of 91 HIV-infected patients aged 18 years and above , currently on ART for at least 12 months and regularly followed-up were consecutively enrolled in the study during January - April 2012. These patients were receiving either first line regimen consisting of 2 NRTIs +1 NNRTI, or second line regimen comprising 2 NRTIs + 1 PI. The choice of a particular regimen was irrespective of potential factors that could induce hyperlactatemia but depended mainly on the availability of ARTs. For quality control purpose, a subset of $30 \mathrm{HIV}$-infected and ART-naïve patients was also recruited. All the participants were confirmed to be positive for HIV antibody through laboratory detection, and the diagnosis was in line with national HIV/AIDS diagnosis criteria. They were free of alcoholism, chronic hepatitis and were also required not to be on plasma lactate modifying therapies at their enrolment. All the procedures used in this study were in accordance with the current revision of the Helsinki Declaration. A Written and signed informed consent was provided by all the subjects. Consent forms and procedures, as well as survey protocol, were approved by the Cameroon National Ethics Committee (Reference number: 234/CNE/SE/2011).

\section{Interview data}

At enrollment standardized data collection forms were completed, including sociodemographic characteristics, medical history (AIDS events, CDC/WHO clinical classification, signs and symptoms of symptomatic hyperlactatemia such as nonspecific gastrointestinal symptoms nausea, vomiting, abdominal pain, discomfort or distension, anorexia, peripheral neuropathy, tiredness, muscle weakness, and dyspnea [24]), laboratory markers (CD4 cell counts, FBS (Fasting Blood Sugar), AST (Aspartate Amino Transferase) and ALT (Alanine Amino Transferase) levels), and antiretroviral used.

\section{Sample collection and biochemical assays}

After at least 12 hours of overnight fasting and a $30 \mathrm{mi}-$ nutes rest, blood was aseptically collected from each participant by venipuncture in a $5 \mathrm{ml}$ EDTA tube, without a tourniquet or fist clenching. Samples were put on ice and immediately transported to the biochemistry laboratory where plasma specimens were separated by centrifugation at $3000 \mathrm{rpm}$ within $5 \mathrm{~min}$ for analyses without delay. Plasma lactate concentrations were determined on an automated clinical chemistry analyzer (BA-88A, MINDRAY laboratory, China) using kits (BIOREX diagnostics, UK) based on the enzymatic conversion of L-lactate to pyruvate by L-lactate oxidase [25].

\section{End point definitions}

Concentrations of plasma lactate less than $3 \mathrm{mmol} / \mathrm{L}$ were defined as normolactatemic, while those equal or above $3 \mathrm{mmol} / \mathrm{L}$, were consider as hyperlactatemic.

\section{Statistical analysis}

Data were coded, entered, and analyzed using SPSS version 20.0 (SPSS Inc., Chicago, Ilinois, USA). Qualitative and quantitative variables were analysed and compared with $\mathbf{X}^{2}$ test and Student's $\mathbf{t}$-test respectively. Results are expressed as proportion or mean \pm SD. The Pearson correlation was used to established the correlation between the variables. ORs with 95\% CIs were used to appreciate the impact of different variables on the occurrence of hyperlactatemia, and were calculated by both univariate and multivariate logistic regression analyses while adjusting for confounders. A $p$ value $<0.05$ was used to characterize significant results.

\section{Results}

A total of 121 patients were included in this study including 91 HIV patients currently taking ART, and 30 ART-naïve HIV patients. Table 1 presents the whole patient clinical characteristics and biochemical determinations. The age ranged from 25 to 67 years old (Mean $=40.3$ ) for patients under ART and between 24 to 64 years old $($ Mean $=38)$ for naive ART patients. All the parameters recorded 
Table 1 Clinical and biochemical characteristics of the study participants

\begin{tabular}{|c|c|c|c|c|}
\hline & \multicolumn{3}{|c|}{ Mean \pm SD } & \multirow[t]{2}{*}{ p } \\
\hline & Patients on ART $(n=91)$ & ART-naïve patients $(n=30)$ & Overall $(n=121)$ & \\
\hline Age (years) & $40.3 \pm 9.9$ & $38.5 \pm 11.8$ & $39.8 \pm 10.4$ & 0.327 \\
\hline Sex (Male/Female) & $22 / 69$ & $6 / 24$ & $28 / 93$ & 0.638 \\
\hline CDC Clinical Classification (A/C) & $90 / 1$ & $30 / 0$ & $120 / 1$ & 0.752 \\
\hline CD4 count (cells/mm³) & $410.4 \pm 231.9$ & $261.8 \pm 173.0$ & $373.2 \pm 226.9$ & 0.192 \\
\hline AST (IU/L) & $27.8 \pm 15.9$ & $25.6 \pm 10.1$ & $27.3 \pm 14.6$ & 0.171 \\
\hline ALT (IU/L) & $25.6 \pm 20.8$ & $23.1 \pm 8.3$ & $24.9 \pm 18.4$ & 0.104 \\
\hline FPG (g/L) & $0.9 \pm 0.2$ & $0.9 \pm 0.2$ & $0.9 \pm 0.2$ & 0.564 \\
\hline LDH (IU/L) & $441.1 \pm 138.1$ & $485.1 \pm 168.6$ & $452.0 \pm 146.8$ & 0.253 \\
\hline Lactataemia (mmol/L) & $2.3 \pm 1.3$ & $1.7 \pm 0.7$ & $2.2 \pm 1.2$ & $0.002^{*}$ \\
\hline Hyperlactatemic patients (\%) & 18.7 & 6.7 & 15.7 & 0.095 \\
\hline
\end{tabular}

FBS: Fasting Blood Sugar; ALT: Alanine Amino Transférase, AST: Aspartate Amino Transférase, LDH: Lactate Dehydrogenase; * $p<0.05$.

were higher in the group of patients on ART therapy compare to ART naïve except the level of LDH. There was no statistically significant difference between the two groups regarding all these parameters except lactatemia which was significantly $(\mathrm{p}<0.005)$ higher in the group of patients on ART (Table 1). Only classes A and $\mathrm{C}$ of the CDC/WHO clinical classification of the HIV infection were noticed. Figure 1 depicts the different therapeutic regimens with the combination AZT 3TC - NVP being the most recorded (32.97\%). The first line ART regimens was the most encountered (66\%). However $25.27 \%$ of the patients were on LVP/r- TDF-3TC (second line regimen). The mean lactataemia was significantly higher in the group of HIV patients currently taking ART than in the ART-naive one $(2.3 \pm 1.3$ and $1.7 \pm 0.7 \mathrm{mmol} / \mathrm{L}$ respectively) (Table 1). Nineteen over the one hundred and twenty one participants (15.70\%) presented with hyperlactatemia; among them 17 were HIV patients currently taking ART (18.7\%) and the 2 others (6.7\%) being ART-naïve patients (Table 1).

Table 2 compares clinical and biochemical parameters between normolactatemic and hyperlactatemic patients taking ART. We did not observe any statistically significant difference between the two groups according to age, sex, CD4+ T-cells count at time of hyperlactatemia, duration of cumulative ART exposure prior to

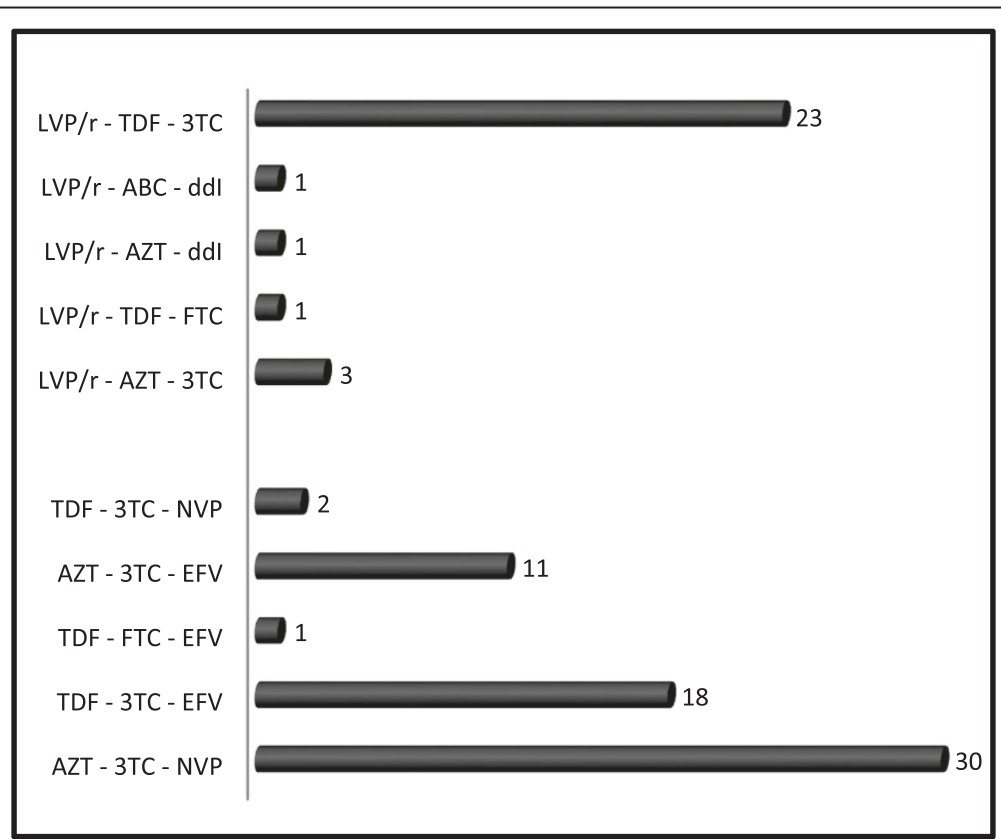

Figure 1 Different therapeutic regimens the 91 ART-receiving subjects were placed on: up are represented second line regimens and down the first line ones and the numbers refer to the number of subjects on each regimen. 
Table 2 Characteristics of patients on ART with regard to the occurrence of hyperlactatemia

\begin{tabular}{|c|c|c|c|}
\hline & $\begin{array}{l}\text { Normolactatemic } n=74 \\
(\text { Mean } \pm \text { SD) }\end{array}$ & $\begin{array}{l}\text { Hyperlactatemic } n=17 \\
(\text { Mean } \pm \text { SD })\end{array}$ & $\mathbf{p}$ \\
\hline Age (years) & $40.3 \pm 9.4$ & $39.9 \pm 12.2$ & 0.865 \\
\hline Sex (Male/Female) & $16 / 58$ & $6 / 11$ & 0.189 \\
\hline CD4 count at time of hyperlactatemia (cells $/ \mathrm{mm}^{3}$ ) & $414.5 \pm 230.8$ & $392.8 \pm 239.6$ & 0.730 \\
\hline Duration of cumulative ART exposure prior to hyperlactatemia (years) & $5.2 \pm 2.9$ & $4.5 \pm 3.2$ & 0.382 \\
\hline \multicolumn{4}{|l|}{ ART regimens } \\
\hline First line & 44 & 16 & $0.007^{*}$ \\
\hline Second line & 30 & 1 & \\
\hline \multicolumn{4}{|l|}{ CDC/WHO Class } \\
\hline A & 73 & 17 & 0.813 \\
\hline C & 1 & 0 & \\
\hline FPG (g/L) & $0.9 \pm 0.1$ & $0.9 \pm 0.2$ & 0.220 \\
\hline AST (IU/L) & $26.7 \pm 12.0$ & $32.8 \pm 27.4$ & 0.396 \\
\hline ALT (IU/L) & $23.1 \pm 11.4$ & $36.6 \pm 41.8$ & 0.221 \\
\hline LDH (IU/L) & $419.7 \pm 107.9$ & $534.3 \pm 207.0$ & $0.040^{*}$ \\
\hline Lactataemia (mmol/L) & $1.8 \pm 0.6$ & $4.2 \pm 1.8$ & $<0.001^{*}$ \\
\hline
\end{tabular}

* $p<0.05$.

hyperlactatemia, FBS, AST and ALT. On contrary, the mean serum LDH (Lactate Dehydrogenase), as well as lactatemia, were significantly higher in the hyperlactatemic group $(\mathrm{p}=0.040$ and $\mathrm{p}<0.001$ respectively). Sixteen patients $(25.8 \%)$ on first line regimens significantly develop hyperlactatemia $(\mathrm{p}=0.007)$. Only one patient on second line regimen (1.1\%) presented with severe hyperlactatemia.

Figure 2 is representative of the regimens that have led to hyperlactatemia. There was no statistically significant difference when comparing the mean plasma lactate levels between these regimens. There was no correlation between lactatemia and age $(\mathrm{r}=0.023, \mathrm{p}=0.825), \mathrm{CD} 4+\mathrm{T}$-cells count $(\mathrm{r}=0.071, \mathrm{p}=0.506)$, FBS $(\mathrm{r}=0.151, \mathrm{p}=0.126)$, and between lactatemia and serum LDH $(r=0.181, \mathrm{p}=0.085)$. On the contrary, there was a positive and significant correlation between lactatemia and AST $(\mathrm{r}=0.439, \mathrm{p}<0.001)$, and ALT ( $\mathrm{r}=0.544, \mathrm{p}<0.001$ ) (Table 3).

While undertaking univariate logistic regression analyses, we did not find any statistically significant association between the occurrence of hyperlactatemia and the sex (OR 2.25, 95\% CI 0.789 - 6.42; p = 0.123), the duration of cumulative ART exposure (OR 2.59, 95\% CI 0.87 - 7.72; $\mathrm{p}=0.089$ ), and between the occurrence of hyperlactatemia and ART exposure (OR 3.22, 95\% CI $0.70-14.93 ; \mathrm{p}=0.134$ ) (Table 4). Nevertheless, this latter association became significant after adjusting for age, sex and duration of ART exposure with a coefficient of

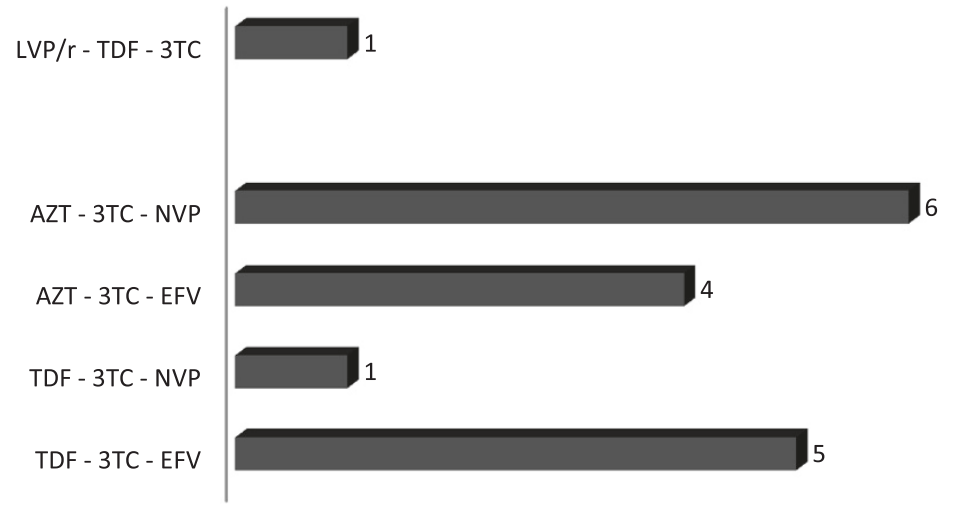

Figure 2 Regimens that have given rise to hyperlactatemia. 
Table 3 Correlation between lactatemia and others parameters

\begin{tabular}{lcc}
\hline Variable & $\mathbf{P}$ & $\begin{array}{c}\text { Pearson's correlation } \\
\text { coefficient }(\mathbf{r})\end{array}$ \\
\hline Age & 0,825 & 0,023 \\
CD4 count & 0,506 & 0,071 \\
Fasting Plasma Glucose & 0,126 & 0,151 \\
LDH & 0,085 & 0,181 \\
AST & $<0,001^{*}$ & $\mathbf{0 , 4 3 9}$ \\
ALT & $<0,001^{*}$ & $\mathbf{0 , 5 4 4}$ \\
\hline
\end{tabular}

${ }^{*} p<0.05$.

Table 4 Factors likely to influence the occurrence of hyperlactatemia

\begin{tabular}{|c|c|c|c|}
\hline & Odds ratio & $95 \% \mathrm{Cl}$ & $\mathbf{p}$ \\
\hline \multicolumn{4}{|l|}{ Sex } \\
\hline Male & 1.00 & & \\
\hline Female & 2.25 & $0.79-6.42$ & 0.130 \\
\hline \multicolumn{4}{|c|}{ Age groups (years) } \\
\hline $20-29$ & 1.00 & & \\
\hline $30-39$ & 0.42 & $0.07-2.57$ & 0.347 \\
\hline $40-49$ & 0.67 & $0.11-4.15$ & 0.664 \\
\hline$\geq \mathbf{5 0}$ & 0.21 & $0.02-2.84$ & 0.243 \\
\hline \multicolumn{4}{|c|}{$\begin{array}{l}\text { Duration of cumulative } \\
\text { ART exposure }\end{array}$} \\
\hline$<5$ years & 1.00 & & \\
\hline$\geq 5$ years & 2.59 & $0.87-7.72$ & 0.089 \\
\hline \multicolumn{4}{|l|}{ ART exposure } \\
\hline No & 1.00 & & \\
\hline Yes & 3.22 & $0.70-14.93$ & 0.134 \\
\hline \multicolumn{4}{|c|}{ CDC clinical stage } \\
\hline A & 1.19 & $1.1-1.28$ & 0.843 \\
\hline $\mathrm{C}$ & 1.00 & & \\
\hline \multicolumn{4}{|l|}{ ART regimen } \\
\hline First line & 10.91 & $1.37-86.70$ & $0.024^{*}$ \\
\hline Second line & 1.00 & & \\
\hline \multicolumn{4}{|c|}{ First line AZT-regimens } \\
\hline No & 1.00 & & \\
\hline Yes & 1.02 & $0.32-3.32$ & 0.969 \\
\hline \multicolumn{4}{|c|}{ First line TDF-regimens } \\
\hline No & 1.00 & & \\
\hline Yes & 1.17 & $0.35-3.97$ & 0.797 \\
\hline \multicolumn{4}{|c|}{ First line 3TC-regimens } \\
\hline No & 1.00 & & \\
\hline Yes & 0.98 & $0.94-1.02$ & 0.742 \\
\hline
\end{tabular}

determination of 0.2 (aOR 5.44, 95\% CI 1.06 - 27.84; $\mathrm{p}=0.042$ ), but after removing the duration of ART exposure from the model, the last-mentioned association was no more significant (aOR 4.09, 95\% CI 0.82 - 20.33; $\mathrm{p}=0.085$ ). Being on a first line ART regimen increased more than 10 times the risk of developing hyperlactatemia (OR 10.91, 95\% CI 1.37 - 86.70; $\mathrm{p}=0.024$ ), this risk becoming more important after adjusting for age, sex, and duration of ART exposure (aOR 16.22, 95\% CI 1.57 - 167.91; $\mathrm{p}=0.019$ ) with a coefficient of determination of 0.32 . We finally observe no significant association between the occurrence of hyperlactatemia and zidovudinecontaining regimens, lamivudine-containing regimens, and with tenofovir-containing regimens (Table 4).

\section{Discussion}

Lactate is the final product of anaerobic glycolysis, which in a normal state does not accumulate in the body. However, under circumstances such as oxygen deprivation of tissue or impaired oxidative phosphorylation, hyperlactatemia occurs. Lactic acidosis in the absence of tissue ischemia is the hallmark of type B lactic acidosis [26]. Among all anti-HIV medications, it is the NRTIs that have been implicated in type B lactic acidosis [26]. In fact, the pharmacologically active triphosphate moieties of NRTIs act as substrates for human mitochondrial DNA-polymerase gamma, thereby having the potential for incorporation into mitochondrial DNA (mtDNA). As well, mitochondrial exonuclease is inefficient at removing the nucleoside triphosphates $[27,28]$. The combination of nucleoside triphosphate incorporation and inadequate removal disrupts mtDNA synthesis and results in the arresting of mtDNA-encoded protein synthesis. Eventually, a disruption of oxidative phosphorylation ensues, and lactate accumulates [2].

Stavudine, zidovudine and didanosine are the most frequent NRTIs incriminated in the occurrence of hyperlactatemia [2,6,9-14,29]. The combination of stavudine and didanosine is associated with the greatest relative risk [24]. In this study we had no patient placed on a stavudine-containing regimens because stavudine has been withdrawn from ART regimens recommended for anti-HIV medications in Cameroon since 2011, mainly due to its dyslipidemic effects proven in this milieu [30] and in accordance with the latest WHO guidelines [14]. We observed in our study that zidovudine, lamivudine and tenofovir were not implicated in the occurrence of hyperlactatemia, probably because of the small sample size and probably, the combination of all these nucleoside analogues would increase risk of adverse event development. These findings concur with those from Marceau et al. [29] where increased lactate was not associated with treatment containing lamivudine, zidovudine, abacavir, zalcitabine, efavirenz, nevirapine, and interferon 
and/or ribavirin. After regrouping regimens in first and second line regimen, we found lactatemia of patients on first line regimens being significantly higher than that of the ones on second line regimen. Furthermore, first line regimen use may confer a sixteen-fold increased risk of developing hyperlactatemia when controlling for other risk factors. These findings can be explained by the fact that PIs, which are part of second line regimen, have not been reported to cause any mitochondrial toxicity [7] given their lack of selectivity for HIV reverse transcriptase [14]. It has been also shown that the incidence and prevalence of hyperlactatemia may vary depending on the number and choice of NRTIs present in an antiretroviral regimen. In fact, in a longitudinal cohort study involving 2144 patients receiving NRTI therapy, the risk of symptomatic hyperlactatemia increased more than 2-fold for each additional NRTI used in a given regimen [31]. Different combinations of NRTIs were also associated with different rates of symptomatic hyperlactatemia [31].

We found significantly elevated plasma lactate levels in patients currently taking ART compared to ARTnaïve ones, with an independent five-fold increased risk for the subjects on ART for developing hyperlactatemia when controlling for other covariates. But contrary to John et al. [7] we did not observe any relationship between the occurrence of hyperlactatemia and duration of cumulative ART exposure (especially stavudine in their case). The Lactic Acidosis International Study group [17], as well as Marceau et al. [32], showed an association of older age (age $>40$ years) with the development of hyperlactatemia. This was not seen in our study nor in some other ones $[7,29,33]$ probably because our study had a little bit younger age distribution than theirs (mean of 40 years vs. 42 and 44 years respectively). Moreover we found no relationship between the female sex and the occurrence of hyperlactatemia. The same results were reported in others studies [7,29,33]. By contrast, some relevant studies have clearly demonstrated that the female sex constitutes a strong independent risk factor for developing hyperlactatemia [17-20,22,23,34,35], even though the reasons for the observed sex differences in rates of toxicity remain uncertain. As shown in other studies, we observed no association between CD4+ T-cells count, CDC/WHO clinical classification and the occurrence of hyperlactatemia $[7,29,33]$.

There is no consensus on the different thresholds for defining normolactatemia, mild to moderate hyperlactatemia (either symptomatic or not), and severe hyperlactatemia. It has been proposed, from a clinical point of view, that clinically relevant hyperlactatemia may be defined as a lactate concentration of $2.25-5 \mathrm{mmol} / \mathrm{L}$, and severe lactic acidosis, as a lactate concentration $>5 \mathrm{mmol} / \mathrm{L}$ with a $\mathrm{pH}<7.3$ or bicarbonate $<20 \mathrm{mmol} / \mathrm{L}$ and clinical signs of multiorgan failure [36]. In their study, Marceau et al. [32], based on a previous determination of the reference interval for plasma lactate obtained from 200 healthy venous blood samples (range: $1.5-2.25 \mathrm{mmol} / \mathrm{L}$; mean \pm SD: $1.4 \pm 0.3 \mathrm{mmol} / \mathrm{L}$ ) [32], defined concentrations of plasma lactate $<2.25 \mathrm{mmol} / \mathrm{L}$ as normolactatemic, those between 2.25 and $5 \mathrm{mmol} / \mathrm{L}$ as moderately increased, and plasma lactate $>5 \mathrm{mmol} / \mathrm{L}$ was considered highly increased. With these definitions, $75.2 \%$ of their patients were normolactatemic, $23 \%$ had moderately increased serum lactate, and $1.8 \%$ had high serum lactate giving thus an overall prevalence of hyperlactatemia (plasma lactate $\geq 2.25 \mathrm{mmol} / \mathrm{L}$ ) of $24.8 \%$ [32]. We had a little bit more normolactatemic subjects than them $(81.3 \%$ vs. $75.2 \%)$ and less hyperlactatemic patients (18.7\% vs. $24.8 \%$ ) assumedly because our definition of normolactatemia was less restrictive than theirs (i.e. $<3 \mathrm{mmol} / \mathrm{L}$ vs. $<$ $2.25 \mathrm{mmol} / \mathrm{L})$. As we, they found that the mean value of plasma lactate was significantly higher in the hyperlactatemic group than in the normolactatemic one [32].

The prevalence of asymptomatic hyperlactatemia reported in resource-rich settings varies from one area to another $8.3 \%$ [33], 8.7\% [37], 18.3\% [7] and 21\% [4]. As a matter of fact, the lack of a standard biochemical definition for lactic acidosis and symptomatic hyperlactatemia causes confusion and affects incidence and prevalence rates.

Nevertheless, it has been hypothesized that a lactate measurement $>5 \mathrm{mmol} / \mathrm{L}$ is defined as severe hyperlactatemia and therefore includes lactic acidosis [17,35]. Fabian et al. in South Africa [35] reported a prevalence of severe hyperlactatemia of $1.3 \%$ corroborating our $1.1 \%$ even though a huge number of their patients were on stavudine-based regimens.

Unfortunately, the cross-sectional design of the present study could not permit us to determine the incidence and time to onset of hyperlactatemia. Menezes et al. [29] reported a median time to the development of lactic acidosis of 10.8 months while Wester et al. [20] reported a median time of 7.5 months. Moreover we were unable to investigate for other risk factors incriminated or not in the development of hyperlactatemia such as higher baseline BMI $\left(>30 \mathrm{~kg} / \mathrm{m}^{2}\right)$, lower initial CD4+ T-cells count, higher baseline serum creatinine levels at ART-initiation, this because these informations were lacking in the majority of our patients' files. It is unclear whether and what extent symptomatic hyperlactatemia might progress to lactic acidosis or whether they are two completely separate disorders [7,37]. In fact, routine monitoring of serum lactate levels does not appear to be warranted [7]. Mild, asymptomatic elevations in lactate levels have not been predictive of progression to lactic acidosis [4,7], and a positive predictive value of only $39 \%$ for the confirmation of lactic acidosis following a single 
elevated value has been reported [37]. Taking into account that lactic acidosis can present quite precipitously and progress in a fulminant manner and those patients can be relatively asymptomatic even with high lactate concentrations, lactate levels should be measured as soon as clinical manifestations suggestive of possible symptomatic hyperlactatemia occur in patients currently taking ART. Marceau et al. [32] have proposed that lactate measurements might be performed every 6 months under standardized conditions in asymptomatic patients to detect a trend of increasing lactate concentrations.

\section{Conclusion}

Our results showed that chronic compensated and moderated hyperlactatemia is common in ART-exposed HIV infected Cameroonians. Even though stavudine is no more part of ART-regimens, being on a first line regimen constitutes a strong and important risk factor for developing hyperlactatemia. Early diagnosis of symptomatic hyperlactatemia by measurement of plasma lactate under standardized conditions may allow safe outpatient care and optimize the management of these HIVinfected patients on ART.

\section{Competing interest}

There is no conflict of interest regarding any of the authors.

\section{Authors' contributions}

Study Concept and design: JNY, VJAM, CTM, CAP, CT. Data collection and performing experiments: CTM, VJAM. Statistical Analysis: JRNN. Drafting: JRNN, CTM, CAP. Manuscript Revision: TCM, JRNN, VJAM, CAP, CT, JNY. Study Supervision: JNY. All authors read and approved the final manuscript.

\section{Acknowledgements}

The authors gratefully acknowledge all the patients who have accepted to take part in this study.

\section{Author details}

${ }^{1}$ Laboratory of Biochemistry, Yaounde University Teaching Hospital, Yaounde, Cameroon. ${ }^{2}$ Department of Biochemistry and Physiological Sciences, University of Yaounde I, Yaounde, Cameroon. ${ }^{3}$ Department of Biochemistry, Faculty of Sciences, University of Yaounde I, Yaounde, Cameroon. ${ }^{4}$ Intensive Care Unit, Mother and Child Centre, Chantal Biya Foundation, Yaounde, Cameroon. ${ }^{5}$ Laboratory of Hematology, Yaounde University Teaching Hospital, Yaounde, Cameroon.

Received: 17 June 2013 Accepted: 10 January 2014 Published: 15 January 2014

\section{References}

1. Palella FJJ, Delaney KM, Moorman AC, Loveless MO, Fuhrer J, Satten GA, et al: Declining morbidity and mortality among patients with advanced immunodeficiency virus infection. HIV Outpatient Study Investigators. N Engl J Med 1998, 338:853-860.

2. Brinkman K, ter Hofstede HJM, Burger DM, Smeitink J, Koopmans PP: Adverse effects of reverse transcriptase inhibitors: mitochondrial toxicity as common pathway. AIDS 1998, 12:1735-1744.

3. Dieterich DT: Long-term complications of nucleoside reverse transcriptase inhibitor therapy. AIDS Reader. 2003, 13:176-178.

4. Vrouenraets SME, Treskes M, Regez RM, et al: Hyperlactatemia in HIV-infected patients: the role of NRTI-treatment. Antivir Ther 2002, 7:239-244.
5. Carr A, Miller J, Law M, Cooper DA: A syndrome of lypoatrophy, lactic acidemia and liver dysfunction associated with HIV nucleoside analogue therapy: contribution to protease inhibitor-related lipodystrophy syndrome. AIDS 2000, 14:F25-F32.

6. Gérard Y, Maulin L, Yazdanpanah Y, De La Tribonniere X, Amiel C, Maurage CA, et al: Symptomatic hyperlactataemia: an emergency complication of antiretroviral therapy. AIDS 2000, 14:2723-2730.

7. John M, Moore CB, James IR, Nolan D, Upton RP, McKinnon EJ, et al: Chronic hyperlactatemia in HIV-infected patients taking antiretroviral therapy. AIDS 2001, 15:717-723.

8. Scalzini A, Tomasoni D, Gattuso G, Fibbia G: Prevalence of asymptomatic hyperlactatemia in HIV patients treated with nucleoside analogues, $6^{\text {th }}$ International Congress on Drug Therapy in HIV infection. Glasgow, UK; 2002:116. Abstract available: http://ww1.aegis.org/conferences/hiv-glasgow/ 2002/hiv6.pdf.

9. Brinkman $K$, Smeitink JA, Romijin JA, Reiss P: Mitochondrial toxicity induced by nucleoside-analogue reverse transcriptase inhibitors is a key factor in the pathogenesis of antiretroviral-therapy-related lipodystrophy. Lancet 1999, 354:1112-1115.

10. Sundar K, Suarez M, Banogon PE, Shapiro JM: Zidovudine induced fatal lactic acidosis and hepatic failure in patients with acquired immunodeficiency syndrome: report of two patients and review of the literature. Crit Care Med 1997, 25:1425-1430.

11. Bissuel F, Bruneel F, Habersetzer F, Chassard D, Cotte L, Chevalier M, et al: Fulminant hepatitis with severe lactate acidosis in HIV-infected patients on didanosine therapy. J Intern Med 1994, 235:337-342.

12. Lonergan JT, Behling C, Pfander H, Hassanein TI, Mathews WC: Hyperlactatemia and hepatic abnormalities in 10 human immunodeficiency virus-infected patients receiving nucleoside analogue combination regimens. Clin Infect Dis 2000, 31:162-166.

13. Delgado J, Harris M, Tesiorowski A, Montaner JSG: Symptomatic elevations of lactic acid and their response to treatment manipulation in human immunodeficiency virus-infected persons: a case series. Clin Infect Dis 2001, 33:2072-2074.

14. World Health Organization: Antiretroviral therapy for HIV infection in adults and adolescents: recommendations for a public health approach-2010 revision. Geneva: World Health Organization; 2010.

15. Murphy RA, Sunpath H, Kuritzkes DR, Venter F, Gandhi RT: Antiretroviral therapy-associated toxicities in the resource-poor world: the challenge of a limited formulary. J Infect Dis 2007, 196:S449-S456.

16. Rosen S, Long L, Fox M, Sanne I: Cost and cost-effectiveness of switching from stavudine to tenofovir in first-line antiretroviral regimens in South Africa. J Acquir Immune Defic Syndr 2008, 48:334-344.

17. Lactic Acidosis International Study Group: Risk factors for lactic acidosis and severe hyperlactataemia in HIV-1-infected adults exposed to antiretroviral therapy. AIDS 2007, 21:2455-2464.

18. Bolhaar MG, Karstaedt AS: A high incidence of lactic acidosis and symptomatic hyperlactatemia in women receiving highly active antiretroviral therapy in Soweto. South Africa. Clin Infect Dis. 2007, 45:254-260

19. Geddes R, Knight S, Moosa MY, Reddi A, Uebel K, Sunpath H: A high incidence of nucleoside reverse transcriptase inhibitor (NRTI)-induced lactic acidosis in HIV-infected patients in a South African context. S Afr Med J 2006, 96:722-724.

20. Wester CW, Okezie OA, Thomas AM, Bussmann H, Moyo S, et al: Higher-than-expected rates of lactic acidosis among highly active antiretroviral therapy-treated women in Botswana: preliminary results from a large randomized clinical trial. J Acquir Immune Defic Syndr 2007, 46:318-322.

21. van Griensven J, Zachariah R, Rasschaert F, Mugabo J, Atte EF, Reid T: Stavudine- and nevirapine-related drug toxicity while on generic fixed-dose antiretroviral treatment: incidence, timing and risk factors in a three-year cohort in Kigali, Rwanda. Trans R Soc Trop Med Hyg 2010, 104:148-153.

22. Boulle A, Orrel C, Kaplan R, Van Cutsem G, McNally M, Hilderbrand K, et al: Substitutions due to antiretroviral toxicity or contraindication in the first 3 years of antiretroviral therapy in a large South African cohort. Antivir Ther 2007, 12:753-760.

23. Osler M, Stead D, Rebe K, Meintjes G, Boulle A: Risk factors for and clinical characteristics of severe hyperlactataemia in patients receiving antiretroviral therapy: a case-control study. HIV Med 2009, 11:121-129. 
24. Ogedegbe AE, Thomas DL, Diehl AM: Hyperlactataemia syndromes associated with HIV therapy. Lancet Infect Dis 2003, 3:329-337.

25. Gutmann I, Wahlefeld A: Lactate measurements in biological fluids. In Methods of enzymatic analysis. 2nd edition. Edited by Bergmeyer HV. New York: Academic Press; 1974:1461-1465.

26. Ritz E: Heidland A. Lactic acidosis. Clin Nephrol. 1977, 7:231-240.

27. Johnson AA, Ray AS, Hanes J, Suo Z, Colacino JM, Anderson KS, et al: Toxicity of antiviral nucleoside analogs and the human mitochondrial DNA polymerase. J Biol Chem 2001, 276:40847-40857.

28. Lim SE, Copeland WC: Differential incorporation and removal of antiviral deoxynucleotides by human DNA polymerase gamma. J Biol Chem 2001, 276:23616-23623.

29. Menezes CN, Maskew M, Sanne I, Crowther NJ, Raal FJ: A longitudinal study of stavudine-associated toxicities in a large cohort of South African HIV infected subjects. BMC Infect Dis 2011, 11:244.

30. Pefura Yone EW, Betyoumin AF, Kengne AP, Kaze Folefack FJ, Ngogang J: First-line antiretroviral therapy and dyslipidemia in people living with HIV-1 in Cameroon: a cross-sectional study. AIDS Res Ther 2011, 8:33.

31. Lonergan JT, Havlir D, Barber E, Mathews WC: Incidence of symptomatic hyperlactatemia in HIV-infected adults on NRTIS, 9th Conference on Retroviruses and Opportunistic Infections. Seattle; 2002. Abstract available: http://www.retroconference.org/2002/Abstract/13202.htm.

32. Marceau G, Sapin V, Jacomet C, Ughetto S, Cormerais L, Regagnon C, et al: Frequency, risk factors, and outcome of hyperlactatemia in HIV-positive persons: implications for the management of treated patients. Clin Chem 2003, 49:1154-1162

33. Boubaker K, Flepp M, Sudre P, Furrer H, Haensel A, Hirschel B, et al: Hyperlactatemia and antiretroviral therapy: the Swiss HIV Cohort Study. Clin Infect Dis 2001, 33:1931-1937.

34. Matthews LT, Giddy J, Ghebremichael M, Hampton J, Guarino AJ, Ewusi A, et al: A risk-factor guided approach to reducing lactic acidosis and hyperlactatemia in patients on antiretroviral therapy. PLoS One 2011, 6:e18736.

35. Fabian J, Venter WD, Mkhabela L, Levin JB, Baker L, Naicker S: Symptomatic hyperlactataemia in adults on antiretroviral therapy: a single-centre experience. S Afr Med J 2008, 98:795-800.

36. Brinkman K: Management of hyperlactatemia: no need for routine lactate measurements. AIDS 2001, 15:795-797.

37. Moyle GJ, Datta D, Mandalia S, Morlese J, Asboe D, Gazzard BG: Hyperlactataemia and lactic acidosis during antiretroviral therapy: relevance, reproducibility and possible risk factors. AIDS 2002, 16:1341-1349.

doi:10.1186/1742-6405-11-2

Cite this article as: Mamiafo et al: Hyperlactatemia in a group of HIV patients living in Yaounde-Cameroon. AIDS Research and Therapy 2014 11:2.

\section{Submit your next manuscript to BioMed Central and take full advantage of:}

- Convenient online submission

- Thorough peer review

- No space constraints or color figure charges

- Immediate publication on acceptance

- Inclusion in PubMed, CAS, Scopus and Google Scholar

- Research which is freely available for redistribution 